\title{
ABOUT THE PECULIARITIES OF USING THE OPTICAL BISTABILITY PHENOMENON IN MEASURING SYSTEMS
}

Claudia Yu. Zenkova

Chernivtsi National University, Optics and Spectroscopy Department

2, Kotsyubynsky Str., Chernivtsi, Ukraine, 58012

\begin{abstract}
The influence of outside factors, such as temperature, polarization of the probing laser beam, the size of the magnetic field, on the formation of the multilevel measuring and controlling system has been analyzed. The use of the optical bistability (OB) phenomenon, as an alternative mechanism for creating such systems, has been proposed. Using layer crystals of the $\mathrm{Pbl}_{2}$-type as an example, we have demonstrated the possibility of operating the development of nonlinear outgoing characteristics with the aim of controlling the size of signals in metrology systems.
\end{abstract}

Keywords: layer crystal, optical bistability, exciton.

\section{INTRODUCTION}

The diversity of laser irradiation power measuring techniques and methods of signal value controlling allow to create multifunctional small-sized devices, which can be used in metrology. This raises the question as to the possibility of controlling small values of space-frequency configurations of the active medium output intensities. Here we are attacking the problem of creating optical bistable devices ${ }^{1}$. The effect of optical bistability can be used both in measuring systems, where instantaneous switching in the mode of strong transmission for a certain period of time is necessary, and in active metrology systems, in which it is necessary to realize the power control of the beam in service by a weak optical inlet signal.

The phenomenon of optical bistability was observed for the first time by McCall as a hysteresis anomaly of laser light transmitting by sodium-laden vapor ${ }^{2}$. Optical bistability is based on the hysteresis dependence of the outcoming intensity on the incoming on $e^{3,4}$. It is very important to choose the medium where the nonlinearity of the inner parameters will manifest itself most distinctly. That is why a layer crystal was chosen. The distinctive property of layer crystals is that pressure constants in layers and between them differ by almost a two-order term. This significantly differentiates them from organic and inorganic semiconductors and dielectrics. The essential anisotropy of physical properties along layers and between them causes anomalous behavior of the vibration spectrum - arising of low-energy optical phonons and bending waves ${ }^{5}(\mathrm{BW})$. The specific interaction of laser radiation with such a medium will result in a complex dynamics of the crystal absorptive power. The formation of nonlinear absorption by layer crystals under the influence of different outside factors is investigated in the works ${ }^{6-8}$.

The aim of this review - is to establish the conditions under which the OB effect can play a determining role in the forming the probing signal of metrological systems.

\section{ABSORPTIVE POWER FORMATION IN LAYER CRYSTALS}

The cited experimental data ${ }^{9}$ present layer crystals as a structure which is sharply anisotropic and which is characterized by a set of exciton states with different energy values and dipole moments. Here the energy of the basic exciton state is determined by the direction of observing the crystal and the light polarization: when the light is propagating in the region of the layer packet $(\vec{k} \perp c, \vec{k}$ presets the polarization azimuth, $\mathrm{c}$ - is the orientation of the crystal crystallographic axis) an exciton state with the smallest energy value is formed $(\vec{k} \perp c, \vec{E} \perp c)$ (according to the measurements ${ }^{10}$ in the reflection spectra for the $\mathrm{Pbl}_{2}$ crystals). Correspondingly, the maximums of other exciton states are presented by the values of the energy intervals: $E_{\vec{k} \| c, \vec{E} \perp c}-E_{\vec{k} \perp c, \vec{E} \perp c}=1,2 \mathrm{meV}, E_{\vec{k} \perp c, \vec{E} \| c}-E_{\vec{k} \| c, \vec{E} \perp c}=0,6 \mathrm{meV}$. A similar energy anisotropy of exciton states was experimentally noted in other layer crystals as well, e.g. $\mathrm{Hgl}_{2}{ }^{11}$. 
Thus, taking into consideration an essential anisotropy of the layer crystal $\mathrm{Pbl}_{2}$, the dependence of its features on the direction of irradiation relative to the crystallographic axis, and placing the crystal into an external magnetic field, we obtain the expression for the absorption coefficient. In this case we use the fundamental factors of the Theory of the Absorption Band Form Function ${ }^{12}$. Then the absorption coefficient is $\quad K(\omega, h)=\frac{2 \pi D_{0}^{2}}{L} \alpha(\omega, h)=\frac{2 \pi}{L} \sum_{i=1}^{3} D_{i}^{2} S_{i}(\omega, h)$

where $D_{1}=D_{0} \sin \varphi \sin \psi, D_{2}=\xi D_{0} \sin \varphi \cos \psi, D_{3}=\eta D_{0} \cos \varphi \sin \psi-$

(2)

are the matrix elements of the exciton-photon interaction operator of each of the zones, $\varphi$ and $\psi-$ are the angles at which vectors $\vec{k}$ and $\vec{E}$ are aligned about the crystallographic axis c, $(0<\varphi \leq \pi / 2$, $0<\psi \leq \pi / 2) ; S_{i}(\omega)$ - is the exciton absorption band form function of the corresponding zone; $\mathrm{D}_{0}$ - is responsible for the oscillator power of crystal transiting into the lower exciton state , $\xi D_{0}$ - into the second state, $\eta D_{0}$ - into the third one, $h=\frac{\delta H^{2}}{L}$. Here and further all the values are normalized to the width of the exciton zone $L=\frac{\pi^{2} \hbar^{2}}{2 m^{*} a^{2}}, \quad a_{e x}, m^{*}, \mu^{*}-$ are the radius, effective mass and the reduced electron mass, $a-$ is the crystal lattice constant. The influence of the magnetic field upon the splitting of the exciton levels is obtained experimentally ${ }^{13}$.

We shall take into consideration the exciton spectrum polarization peculiarities with allowance made for two possible energy relaxation mechanisms of exciton excitation - nonlinear interaction with bending waves, whose frequency is $\left(\Omega_{\vec{q}}=\Omega_{2} q^{2}\right)$ and linear interaction with optical phonons $\left(\Omega_{\bar{q}}=\Omega_{1}\right)$ (here $\vec{q}$ is the phonon vector $)^{6-8,14}$.

$$
S_{i}(\omega, h)=\frac{\Gamma_{i}}{\left(\omega-\omega_{0 i}-\Delta_{i}-h\right)^{2}+\Gamma_{i}^{2}} \quad(i=1,2,3)-
$$

is the absorption band form function of each of the exciton excitation zones.

According to the Green function method ${ }^{4}$, which is used for defining the form function of the absorption band $S_{i}(\omega)$ we define the mass operator of the system $M_{i}=\Delta_{i}+i \Gamma_{i}$, where $M_{i}(\omega, h)=M_{1 i}(\omega, h)+M_{2 i}(\omega, h)$, and

$$
\begin{gathered}
M_{1 i}(\omega, h)=\frac{1}{N \hbar^{2}} \sum_{\vec{q}} V_{1}^{2}(\vec{q})\left(\frac{1+n_{\vec{q}}+N_{\vec{q}}}{\omega-\omega_{\vec{q}}-M_{2 i}(\omega, h)-\Omega_{\vec{q}}-h+i \eta}+\frac{n_{\vec{q}}-N_{\vec{q}}}{\omega-\omega_{\vec{q}}-M_{2 i}(\omega, h)+\Omega_{\vec{q}}-h+i \eta}\right), \\
M_{2 i}(\omega, h)=\frac{1}{N \hbar} \sum_{\vec{q}} V_{2}(\vec{q},-\vec{q})\left(1+2 n_{\vec{q}}\right),
\end{gathered}
$$

The constants $V_{1}, V_{2}$ - are constants of the nonlinear interaction of excitons with bending waves and of the linear interaction with optical phonons correspondingly. Here $n_{\vec{q}}=\left[\exp \left(\frac{\hbar \Omega_{\vec{q}}}{k_{B} T}\right)-1\right]^{-1}$, $N_{\vec{k}}=\left[\exp \left(\frac{\hbar \omega_{\vec{k}}-\mu}{k_{B} T}\right)-1\right]^{-1}$ are occupation numbers of phonon and exciton states, the exciton chemical potential $\mu$ defines the exciton concentration at temperature $T$. The calculation of the absorption power was performed for the $2 \mathrm{H}$-polytype $\mathrm{Pbl}_{2}$ layer crystal with the following parameters ${ }^{11} a=4,557 \AA, \Omega_{1}=$ $120 \mathrm{~cm}^{-1}, \Omega_{2}=60 \mathrm{~cm}^{-1}, \varepsilon=11,1, m_{e} \|=1,12 m_{0}, m_{h}^{\|}=0,22 m_{0}$ and also for the model parameters: $L^{\| 1}=10^{4}$ $\mathrm{CM}^{-1}, \Gamma_{0}{ }^{\|}=4,5 \mathrm{~cm}^{-1}, F_{0}{ }^{\|}=0,35, \xi=0,7, \eta=0,8$ for different conditions of crystal irradiation. The results of calculating the layer crystal absorption spectrum in the exciton resonance region allows us to speak about 
the structurization of the absorption curve with a complex form changing dynamics. An important role is also played by the extend of exciton and specific bending waves coupling ${ }^{14}$.

The analysis performed in the works ${ }^{7,8,15}$ show an essential dependence of absorption not only on the frequency and intensity of the external absorption, but on the direction of the optical wave incidence relative to the crystal axis as well. External conditions, namely, the temperature and magnetic field allow to display the particularities of changing the absorption maximum of frequency localization and the peculiarities of controlling the frequency region of reproducing absorption through the size of the magnetic field.

\section{OPTICAL BISTABILITY PHENOMENON}

The effect of laser radiation is many-sided. One of its manifestations is the change of crystal light transmission conditions. A nonlinear response can be expected instead of the linear one. The character of that nonlinear response is essentially determined by the polarization plane direction of the incident radiation relative to the layer crystal axis ${ }^{14}$ or temperature. The arising of exciton concentration and the nonlinear process of their relaxation both on bending waves and optical phonons lead to the formation of cavityless $\mathrm{OB}$ in the exciton resonance region. This fact was repeatedly mentioned in our works ${ }^{6}$. This state can be described mathematically by an exciton balance equation

$N=I_{0} \cdot S(\omega, N, T, h)$,

which, in view of the Law of Buguer, allows us to define the transmission by the crystal $\tau$ with a welldefined input intensity $I_{0}$. We shall cite some results of $O B$ calculations considering the influence of temperature, magnetic field and the excitation of different exciton zones.

\section{a) Temperature $\mathrm{OB}$}

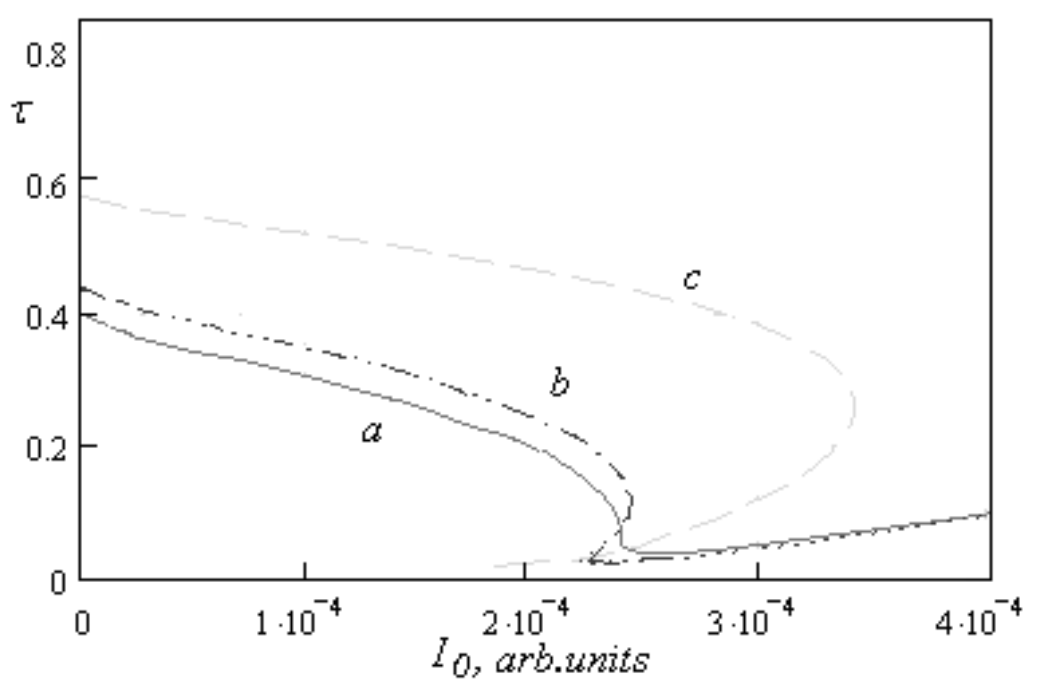

Figure 1 shows the results of the $O B$ calculations for different temperatures and irradiation intensity $\mathrm{I}_{0}=20 \mathrm{~W} / \mathrm{cm}^{2}$, when exciting exciton zones one after another. The exciton zone with the lowest energy is taken as an example in this case. By fixing the registered frequency of crystal excitation we obtain a rather narrow region of temperature $\mathrm{OB}$, whose control is performed by inner factors, namely, by the quantity of excited excitons (assigned by the irradiation intensity) with their subsequent relaxations both on the banding waves and on optical phonons (the temperature effect).

Fig. 1. Temperature optical bistability $(\Delta T=9 \mathrm{~K})$ : a) $T_{\uparrow}=43 \mathrm{~K}$, b) $T=45 \mathrm{~K}$, c) $T_{\downarrow}=53 \mathrm{~K}$.

\section{b) Polarization OB}

All three exciton zones (Exp.1) can arise in the absorption spectrum with the change of the interaction constant and polarization azimuth of the incident wave. Thus, setting the parameters of the model and taking into account the relaxation of the exciton excitation energy on bending waves, we receive the structurized picture of absorption. With $\varphi=\pi / 2, \psi=\pi / 2$ transitions into the first (the lowest) exciton zone take place (Fig.2, curve b); with $\varphi=\pi / 2$, transitions into the second exciton zone take place, and with $\varphi=0, \psi=\pi / 2$ - transitions into the third exciton zone take place (curve $\mathrm{c}$ ). The intermediate values of angles of incidence determine the spectrum with a complex dynamic, where the first band is formed 
by two exciton zones (which practically superpose, because of the minor energy interval between the excitation state), and the second band is formed by the third exciton zone. Thus, the unidirectional vectors $\vec{k}$ and $\vec{c}$, and the subtractive sign of the bond function determine the increase of the interval of the incoming signal intensity $\Delta I_{0 s}$ with the increase of the bond-function value, where the phenomenon of change-over (strong $\tau_{\uparrow}$ - weak $\tau_{\downarrow}$ transmission and vice versa) are essentially actual. The irradiating condition along the layer packet $(\vec{k} \perp c)$ forms the perspective for controlling the outgoing transmission up to $70 \%$ of the incident light (curve b), which practically reaches the limits of transmission for some types of transparent optical materials for industrial use. The rotation of the incident wave polarization plane around the crystallographic axis prescribes the specific character of the dynamics of crystal transmission variations: a distinct depolarization of the absorption band (the excitation of bending waves in different directions of the irradiation wave) causes maximum crystal transmission (up to $80 \%$ ) with the greatest region of controlled bistable signal intensities. Evidently, it is due to the essential activization of the bending wave type phonon modes and the increase of their influence on polarization optical bistability.

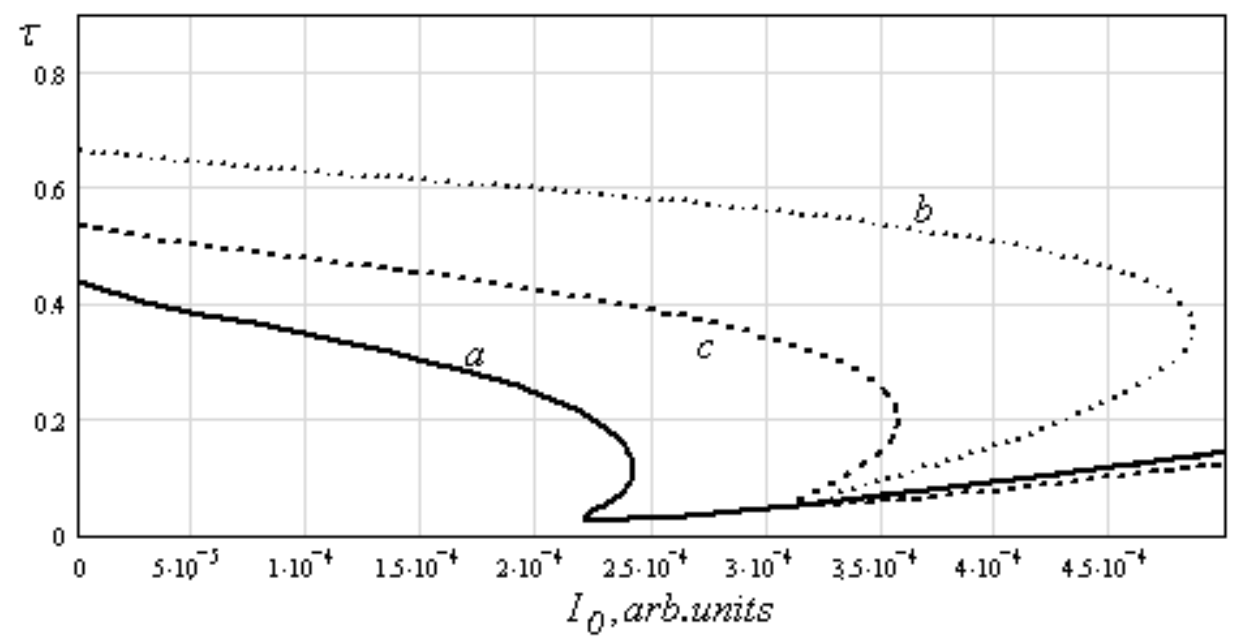

Fig.2. The polarization optical bistability:
a) $\varphi=\pi / 2, \psi=0$,
b) $\varphi=\pi / 2, \psi=\pi / 2$,
c) $\varphi=0, \psi=\pi / 2$

\section{c) Magnetooptical bistability}

We impose the external magnetic field on the investigated crystal. We take into consideration the possibility of exciting different exciton zones by changing the direction of irradiating the crystal layers. In this case we fix the outside factors: the temperature, $T=45 \mathrm{~K}$, the laser irradiation frequency and the corresponding external intensity. An interesting result of defining the magnetooptical response is its dependence on the bending wave excitation conditions. The irradiation of a crystal along its axis $\left(\varphi=0, \psi=\frac{\pi}{2}\right)$ produces an interval between bistable values of the magnetic field $\Delta h_{s}=h_{\uparrow}-h_{\downarrow}=0.00005$. The excitation of the lowest exciton zone $\left(\varphi=\psi=\frac{\pi}{2}\right)$ (the irradiation is perpendicular to the crystal axis c) defines the interval between the bistable values $\Delta h_{s}=0.000117$. The second exciton zone (with crystal excitation under the following conditions $\varphi=\frac{\pi}{2}, \psi=0$ ) presets the interval of controlled values of the magnetic field, where the bistable response can manifest itself, $\Delta h_{s}=0.00025$. In this case the interval is larger than under other irradiation conditions, although it is still rather narrow. The fact can be explained by the external magnetic field-dependence of the intrinsic magnetic moments of the layer packet oscillations. This phenomenon changes the relaxation dynamics of the processes when forming the crystal response. It is important to keep in mind that the absolute value of the magnetic-optical bistability does not depend on the irradiation frequency. The frequency change $\Delta \omega$ allows to obtain a similar bistable response by changing the magnetic field value $\Delta h$. A complete control of the optical bistability realization region is realized by magnetic fields in this case. The analysis of layer crystal magnetic optical bistability at the excitation of different exciton zones show the unique possibility to control the hysteresis loop value and the upper and lower stable state values by changing the size of the magnetic field and, reasonably, the crystal irradiation conditions (fig. 3). In this case there 
is no need to change the radiator for obtaining bistability. It is particular promising for obtaining bistable phenomena in the region of exciton resonances. The availability of strong or weak magnetic fields makes possible to produce a bistable response in a layer crystal, which is very actual for multilevel metrological systems. Layer crystals provide a complete correlation between the magnetic intensity and the bistable frequency region. When imposing an external magnetic field we exercise an additional control over any of the above named factors. Thus, a layer crystal itself, owing to the great diversity of its properties, can serve as a perspective material for creating small easy-to-control elements in the measuring systems.

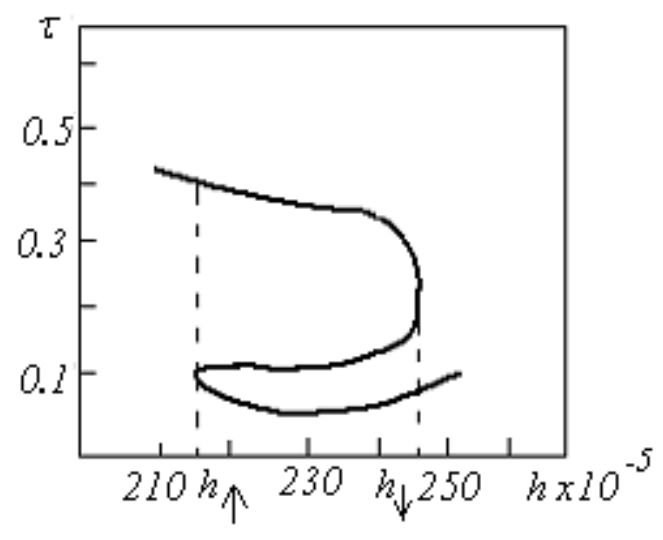

a)

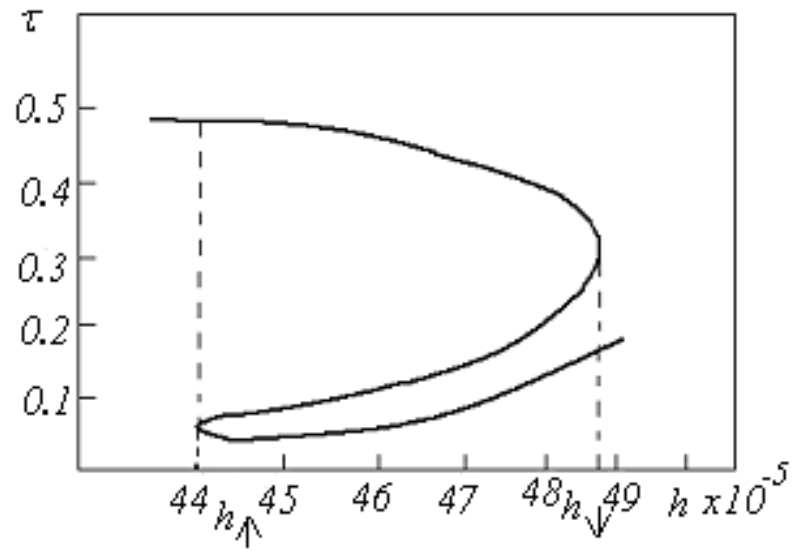

b)

Fig. 3. Magnetooptical bistability ( $a$ - is the excitation of the first exciton zone $\left(\frac{\pi}{2}, 0\right), b-$ is the excitation of the second exciton zone $\left.\left(0, \frac{\pi}{2}\right)\right)$

\section{REFERENCES}

1. H. M. Gibbs, Optical bistability: Controlling Light with Light, N-Y, Acad. Press, 512 p., 1985.

2. S.L. McCall, H.M. Gibbs, G.G. Churchill, T.N.K. Venkatesan, "Optical nonlinearity in the sodium vapour", Bull. Am. Phys. Soc. 20, pp. 636-641, 1975.

3. E. Abraham, C.N. Seaton, S.D. Smith, "The optical computer", Scientific American. 248, pp. 63-71, 1983.

4. B.M.Nitsowich, C.Yu.Zenkova, "The Theory of Optical Bistability in the Region of Exciton Resonances", Optical Engineering. 34, pp.1072-1078, 1995.

5. M.S. Brodin, I.V.Blonskii, Exciton processes in layer crystals, Kyjv, Naukova dumka, 320 p., 1986.

6. C.Yu. Zenkova, V.M. Kramar, N.K. Kramar, "Polarization optical bistability in layer crystals", Proc. of the Symp. Photonics Technol. (Wroclaw, Poland, 2006) pp. 254-257, 2006.

7. C.Yu. Zenkova, V.M. Kramar, N.K. Kramar, O.V. Derevyanchuk, "Optical bistability of layer crystal in the exciton absorption region”, Optics and Spectroscopy. 101, №5, pp.789-794, 2006.

8. C.Yu. Zenkova, "About the possibility of using optical bistability effect in metrology systems", Proc. SPIE. 6616, 2007.

9. R. Minde, G. Ottaviani,C. Canaly C., "Charge Transport in Layer Semiconductors", J. Phys. Chem. Sol. 37, № 2, pp. 417-424, 1976.

10. B.M.Nitsovich, „The electron-phonon interaction in Layer Crystals“, Physics of Solid State. 28, № 8, pp. 2424-2427, 1986 (in Russian).

11. K. Kansaki , I. Imai, "The optical properties of Hgl ${ }_{2}$ ", J. Phys. Soc. Jap. 32, № 4, pp.1003-1009, 1972.

12. B.M. Nitsovich, C.Yu. Zenkova, "The polarization anomaly of exciton spectra", Physics of Solid State.

38, № 5, pp. 1626-1628, 1996.

13. Evans B.L., Young P.A. "Exciton spectra in thin crystals: the diamagnetic effect", Proc.Phys.Soc. 91, pp.475-482, 1967.

14. M.S.Brodin , I.V.Blonskii , B.M.Nitsowich, „Anisotropic exiton scattering by bending waves in layer crystals", Phys. stat. sol. (b). 117, № 2, pp. K99-K103, 1983.

15. C. Zenkova, V. Kramar, N.Kramar, "Magnetooptical bistability of layer crystals", Proc. SPIE. 7008, 2008. 